Discrete Comput Geom 32:129-139 (2004)

DOI: $10.1007 / \mathrm{s} 00454-003-0834-3$

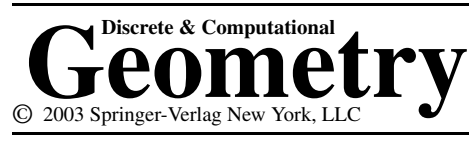

\title{
The Integer Hull of a Convex Rational Polytope
}

Jean B. Lasserre

LAAS-CNRS, 7 Avenue du Colonel Roche,

31077 Toulouse cédex 4, France

lasserre@laas.fr

\begin{abstract}
Given $A \in \mathbb{Z}^{m \times n}$ and $b \in \mathbb{Z}^{m}$, we consider the integer program $\max \left\{c^{\prime} x \mid A x=\right.$ $\left.b ; x \in \mathbb{N}^{n}\right\}$ and provide an equivalent and explicit linear program $\max \left\{\widehat{c}^{\prime} q \mid \mathrm{M} q=r ; q \geq 0\right\}$, where $\mathrm{M}, r, \widehat{c}$ are easily obtained from $A, b, c$ with no calculation. We also provide an explicit algebraic characterization of the integer hull of the convex polytope $\mathrm{P}=\{x \in$ $\left.\mathbb{R}^{n} \mid A x=b ; x \geq 0\right\}$. All strong valid inequalities can be obtained from the generators of a convex cone whose definition is explicit in terms of $\mathrm{M}$.
\end{abstract}

\section{Introduction}

Let $A \in \mathbb{Z}^{m \times n}, b \in \mathbb{Z}^{m}, c \in \mathbb{R}^{n}$ and consider the integer program

$$
\mathbb{P} \rightarrow:=\max \left\{c^{\prime} x \mid A x=b ; x \in \mathbb{N}^{n}\right\},
$$

where the convex polyhedron $\mathrm{P}:=\left\{x \in \mathbb{R}^{n} \mid A x=b ; x \geq 0\right\}$ is compact. If $\mathrm{P}_{1}$ denotes the integer hull of $\mathrm{P}$, then solving $\mathbb{P}$ is equivalent to solving the linear program $\max \left\{c^{\prime} x \mid x \in \mathrm{P}_{1}\right\}$.

However, finding the integer hull $\mathrm{P}_{1}$ of $\mathrm{P}$ is a difficult problem. As mentioned on $\mathrm{p}$. 15 in [8], and to the best of our knowledge, no explicit (or "simple") characterization (or description) of $\mathrm{P}_{1}$ has been provided so far. In the general cutting plane methods originated by Gomory and Chvátal in the early sixties, and the lift-and-project methods described in, e.g., [4], one obtains $\mathrm{P}_{1}$ as the final iterate of a finite nested sequence $\mathrm{P} \supseteq \mathrm{P}^{\prime} \supseteq \mathrm{P}^{\prime \prime} \supseteq \cdots \supseteq \mathrm{P}_{1}$ of polyhedra. However, in all those procedures, $\mathrm{P}_{1}$ has no explicit description in terms of the initial data $A, b$. On the other hand, for specific polytopes $\mathrm{P}$, one is often able to provide some strong valid inequalities in explicit form, but very rarely all of them (as for the matching polytope of a graph). For more details the interested reader is referred to [1], [2], [4], [6], [7, Section 23], [8, Sections 8 and 9], and the many references therein. 
Contribution. The main goal of this paper is to provide a structural result on the integer hull $\mathrm{P}_{1}$ of a convex rational polytope $\mathrm{P}$, in the sense that we obtain an explicit algebraic characterization of the defining hyperplanes of $\mathrm{P}_{1}$, in terms of generators of a convex cone $C$ which is itself described directly from the initial data $A$, with no calculation.

We first show that the integer program $\mathbb{P}$ is equivalent to a linear program in the explicit form

$$
\max _{q \in \mathbb{R}^{s}}\left\{\widehat{c}^{\prime} q \mid \mathrm{M} q=r ; q \geq 0\right\} .
$$

By explicit we mean that the data $\mathrm{M}, r, \widehat{c}$ of the linear program (1.2) are constructed explicitly and easily from the initial data $A, b, c$. In particular, no calculation is needed and $\mathrm{M}, r$ have all their entries in $\{0, \pm 1\}$. In addition $\mathrm{M}$ is very sparse. Of course, and as expected, the dimension of the matrix $\mathrm{M}$ is in general exponential in the problem size. However, for the class of problems where $A$ has nonnegative integral entries, and $b$ and the column sums of $A$ are bounded, then (1.2) is solvable in time polynomial in the problem size.

There is a simple linear relation $x=\mathrm{E} q$ linking $x$ and $q$, but $q$ is not a lifting of $x$ like in the the lift-and-project procedures described in [4]. It is more appropriate to say that $q$ is a disaggregation of $x$, as will become clear in what follows. Moreover, with each extreme point $q$ of the convex polyhedron $\Omega:=\left\{q \in \mathbb{R}^{s} \mid \mathrm{M} q=r, q \geq 0\right\}$ is associated an integral point $x=\mathrm{E} q$ of $\mathrm{P}$ (i.e., $x \in \mathrm{P} \cap \mathbb{Z}^{n}$ ).

Using the latter result, and when $\mathrm{P}$ is compact, we provide the integer hull $\mathrm{P}_{1}$ in the explicit form $\left\{x \in \mathbb{R}^{n} \mid U x \geq u\right\}$ for some matrix $U$ and vector $u$. By this we mean that $U, u$ are obtained from the generators of a convex cone $C$ which has a very simple and explicit description in terms of $A$ (via $\mathrm{M}$ ). Hence, all strong valid inequalities for $\mathrm{P}_{1}$ can be obtained from the generators of the cone $C$. Of course, in view of the potentially large size of $\mathrm{M}$, one cannot expect to get all generators of $C$ in general. However, we hope that this structural result on the characterization of $\mathrm{P}_{1}$ will be helpful in either deriving strong valid inequalities or validating some candidates inequalities, at least for some specific polytopes P.

\section{Notation and Preliminary Results}

Let $\mathbb{N}$ denote the natural numbers or, equivalently, $\mathbb{Z}_{+}$. For a vector $b \in \mathbb{R}^{m}$ and a matrix $A \in \mathbb{R}^{m \times n}$, denote by $b^{\prime}$ and $A^{\prime} \in \mathbb{R}^{n \times m}$ their respective transpose. Denote by $e_{m} \in \mathbb{R}^{m}$ the vector with all entries equal to 1 . Let $\mathbb{R}\left[x_{1}, \ldots, x_{n}\right]$ be the ring of real-valued polynomials in the variables $x_{1}, \ldots, x_{n}$. A polynomial $f \in \mathbb{R}\left[x_{1}, \ldots, x_{n}\right]$ is written

$$
x \mapsto f(x)=\sum_{\alpha \in \mathbb{N}^{n}} f_{\alpha} x^{\alpha}=\sum_{\alpha \in \mathbb{N}^{n}} f_{\alpha} x_{1}^{\alpha_{1}} \cdots x_{n}^{\alpha_{n}},
$$

for finitely many real coefficients $\left\{f_{\alpha}\right\}$, in the (usual) basis of monomials.

Given a matrix $A \in \mathbb{Z}^{m \times n}$, let $A_{j} \in \mathbb{Z}^{m}$ denote its $j$ th column (equivalently, the $j$ th row of $A^{\prime}$ ); then $z^{A_{j}}$ stands for

$$
z^{A_{j}}:=z_{1}^{A_{1 j}} \cdots z_{m}^{A_{m j}}=\mathrm{e}^{\left\langle A_{j}, \ln z\right\rangle}=\mathrm{e}^{\left(A^{\prime} \ln z\right)_{j}},
$$

and if $A_{j} \in \mathbb{N}^{m}$, then $z^{A_{j}}$ is a monomial of $\mathbb{R}\left[z_{1}, \ldots, z_{m}\right]$. 


\subsection{Preliminary Result}

We first recall the following result:

Theorem 2.1 (A Discrete Farkas Lemma). Let $A \in \mathbb{N}^{m \times n}, b \in \mathbb{N}^{m}$. Then the following two statements are equivalent:

(i) The linear system $A x=b$ has a solution $x \in \mathbb{N}^{n}$.

(ii) The real-valued polynomial $z \mapsto z^{b}-1:=z_{1}^{b_{1}} \cdots z_{m}^{b_{m}}-1$ can be written

$$
z^{b}-1=\sum_{j=1}^{n} Q_{j}(z)\left(z^{A_{j}}-1\right)
$$

for some real-valued polynomials $Q_{j} \in \mathbb{R}\left[z_{1}, \ldots, z_{m}\right], j=1, \ldots, n$, all of them with nonnegative coefficients.

In addition, the degree of the $Q_{j}$ 's in (2.1) is bounded by

$$
b^{*}:=\sum_{j=1}^{m} b_{j}-\min _{k} \sum_{j=1}^{m} A_{j k} .
$$

A proof based on counting techniques via generating functions and inverse $\mathbb{Z}$-transform can be found in [3]. However, thanks to an anonymous referee's remark, a self-contained and simpler proof is provided in Section 2.3 below. First, we make some useful remarks and introduce some additional material.

\subsection{Discussion}

(a) With $b^{*}$ as in (2.2) denote by

$$
s:=s\left(b^{*}\right):=\left(\begin{array}{c}
m+b^{*} \\
b^{*}
\end{array}\right)
$$

the dimension of the vector space of polynomials of degree $b^{*}$ in $m$ variables. In view of Theorem 2.1, and given $b \in \mathbb{N}^{m}$, checking the existence of a solution $x \in \mathbb{N}^{n}$ to $A x=b$ reduces to checking whether or not there exists a nonnegative solution $q$ to a system of linear equations

$$
\mathrm{M} q=r, \quad q \geq 0,
$$

for some matrix $\mathrm{M} \in \mathbb{Z}^{p \times n s}$, and vector $r \in \mathbb{Z}^{p}$, with

- $n s$ variables $\left\{q_{j \alpha}\right\}$, the nonnegative coefficients of the $Q_{j}$ 's,

- $p$ equations to identify the terms of the same power in both sides of (2.1); obviously one has

$$
p \leq s\left(b^{*}+a\right):=\left(\begin{array}{c}
m+b^{*}+a \\
b^{*}+a
\end{array}\right) \quad\left(\text { with } a:=\max _{k} \sum_{j=1}^{m} A_{j k}\right) .
$$

In fact we may and will take $p=s\left(b^{*}+a\right)$. 
This in turn reduces to solving a linear programming (LP) problem. Observe that in view of (2.1), the matrix of constraints $M \in \mathbb{Z}^{p \times n s}$, which has only 0 and \pm 1 coefficients, is easily deduced from $A$ with no calculation (and is very sparse). The same is true for $r \in \mathbb{Z}^{p}$ which has only two nonzero entries (equal to -1 and 1 ).

(b) In fact, from the proof of Theorem 2.1, it follows that one may even enforce the weights $Q_{j}$ in (2.1) to be polynomials in $\mathbb{Z}\left[z_{1}, \ldots, z_{m}\right]$ (instead of $\mathbb{R}\left[z_{1}, \ldots, z_{m}\right]$ ) with nonnegative coefficients (and even with coefficients in $\{0,1\}$ ). However, (a) above shows that the strength of Theorem 2.1 is precisely in allowing $Q_{j} \in \mathbb{R}\left[z_{1}, \ldots, z_{m}\right]$ as it permits to check feasibility by solving a (continuous) linear program. Enforcing $Q_{j} \in \mathbb{Z}\left[z_{1}, \ldots, z_{m}\right]$ would result in an integer program of size larger than that of the original problem.

(c) Theorem 2.1 reduces the issue of the existence of a solution $x \in \mathbb{N}^{n}$ to a particular ideal membership problem, that is, $A x=b$ has a solution $x \in \mathbb{N}^{n}$ if and only if the polynomial $z^{b}-1$ belongs to the binomial ideal $I=\left\langle z^{A_{j}}-1\right\rangle_{j=1, \ldots, n} \subset \mathbb{R}\left[z_{1}, \ldots, z_{m}\right]$ and for some weights $Q_{j}$ all with nonnegative coefficients. In fact, one could prove Theorem 2.1 by an appropriate reduction of the initial problem of the existence of a solution $x \in \mathbb{N}^{n}$ to $A x=b$, to a polynomial ideal membership problem (with special features) in the framework developed in Section 3 of [5], another alternative to the proof in [3].

Next, with $A \in \mathbb{N}^{m \times n}, b \in \mathbb{N}^{m}$, let $\mathrm{P} \subset \mathbb{R}^{n}$ be the convex polyhedron

$$
\mathrm{P}:=\left\{x \in \mathbb{R}^{n} \mid A x=b ; x \geq 0\right\} .
$$

Similarly, with $\mathrm{M} \in \mathbb{Z}^{p \times n s}, r \in \mathbb{Z}^{p}$ as in (2.3), let

$$
\Omega:=\left\{q \in \mathbb{R}^{n s} \mid \mathrm{M} q=r ; q \geq 0\right\}
$$

be the convex polyhedron of feasible solutions $q \in \mathbb{R}^{n s}$ of (2.3). So, obviously, (2.1) holds if and only if $\Omega \neq \emptyset$.

Define the row vector $e_{s}:=(1, \ldots, 1) \in \mathbb{R}^{s}$ and let $\mathrm{E} \in \mathbb{N}^{n \times n s}$ be the block diagonal matrix, whose each diagonal block is the row vector $e_{s}$, that is,

$$
\mathrm{E}:=\left[\begin{array}{cccc}
e_{s} & 0 & \cdots & 0 \\
0 & e_{s} & 0 & \cdots \\
\cdots & \cdots & \cdots & \cdots \\
0 & \cdots & 0 & e_{s}
\end{array}\right] .
$$

Proposition 2.2. Let $A \in \mathbb{N}^{m \times n}, b \in \mathbb{N}^{m}$ be given and let $\mathrm{M}$ be as in (1.2). Let $\mathrm{P}, \Omega$ be the convex polyhedra defined in (2.4) and (2.5).

(a) Let $q \in \Omega$. Then $x:=\mathrm{E} q \in \mathrm{P}$. In particular, if $q \in \Omega \cap \mathbb{Z}^{n s}$, then $x \in \mathrm{P} \cap \mathbb{Z}^{n}$.

(b) Let $x \in \mathrm{P} \cap \mathbb{Z}^{n}$. Then $x=\mathrm{E} q$ for some $q \in \Omega \cap \mathbb{Z}^{n s}$.

(c) The matrix $\mathrm{M}$ is totally unimodular.

(d) Whenever $\Omega \neq \emptyset$, each vertex of $\Omega$ is integral.

Proof. (a) With $q \in \Omega$, let $\left\{Q_{j}\right\}_{j=1}^{n} \subset \mathbb{R}\left[z_{1}, \ldots, z_{m}\right]$ be the set of polynomials (with vector of nonnegative coefficients $q$ ) which satisfy (2.1). Taking the derivative of both 
sides of (2.1) with respect to $z_{k}$, at the point $z=(1, \ldots, 1)$, yields

$$
b_{k}=\sum_{j=1}^{n} Q_{j}(1, \ldots, 1) A_{k j}=\sum_{j=1}^{n} A_{k j} x_{j}, \quad k=1, \ldots, n,
$$

with $x_{j}:=Q_{j}(1, \ldots, 1)$ for all $j=1, \ldots, n$. Next, use the facts that (i) all the $Q_{j}$ 's have nonnegative coefficients $\left\{q_{j \alpha}\right\}$, and (ii) $Q_{j}(1, \ldots, 1)=\sum_{\alpha \in \mathbb{N}^{m}} q_{j \alpha}=(\mathrm{E} q)_{j}$ for all $j=1, \ldots, n$, to obtain $x:=\mathrm{E} q \in \mathrm{P}$. Moreover, if $q \in \Omega \cap \mathbb{Z}^{n s}$, then obviously $x \in \mathrm{P} \cap \mathbb{Z}^{n}$.

(b) Let $x \in \mathrm{P} \cap \mathbb{Z}^{n}$ so that $x \in \mathbb{N}^{n}$ and $A x=b$; write

$$
z^{b}-1=z^{A_{1} x_{1}}-1+z^{A_{1} x_{1}}\left(z^{A_{2} x_{2}}-1\right)+\cdots+z^{\sum_{j=1}^{n-1} A_{j} x_{j}}\left(z^{A_{n} x_{n}}-1\right)
$$

and, whenever $x_{j} \neq 0$,

$$
z^{A_{j} x_{j}}-1=\left(z^{A_{j}}-1\right)\left[1+z^{A_{j}}+\cdots+z^{A_{j}\left(x_{j}-1\right)}\right], \quad j=1, \ldots, n,
$$

to obtain (2.1) with

$$
z \mapsto Q_{j}(z):=z^{\sum_{k=1}^{j-1} A_{k} x_{k}}\left[1+z^{A_{j}}+\cdots+z^{A_{j}\left(x_{j}-1\right)}\right],
$$

and $Q_{j} \equiv 0$ if $x_{j}=0, j=1, \ldots, n$. We immediately see that each $Q_{j}$ has all its coefficients $\left\{q_{j \alpha}\right\}$ nonnegative (and even in $\{0,1\}$ ). Moreover, $Q_{j}(1, \ldots, 1)=x_{j}$ for all $j=1, \ldots, n$, or, equivalently, $x=\mathrm{E} q$ with $q \in \Omega \cap \mathbb{Z}^{n s}$.

(c) That $\mathrm{M}$ is totally unimodular follows from the fact that $\mathrm{M}$ is a network matrix, that is, a matrix with $\{0, \pm 1\}$ entries and with exactly two nonzero entries 1 and -1 in each column (see p. 274 of [7]). Indeed, from the identity (2.1), and the definition of $\mathrm{M}$, each row of $\mathrm{M}$ is associated with a monomial $z^{\alpha}$, with $\sum_{j} \alpha_{j} \leq b^{*}+a$. Thus, consider a particular column of $\mathrm{M}$ associated with the variable $q_{k \alpha}$ (the coefficient of the monomial $z^{\alpha}$ of the polynomial $Q_{k}$ in (2.1), with $\sum_{j} \alpha_{j} \leq b^{*}$ ). From (2.1), the variable $q_{k \alpha}$ is involved only:

- in the row (or equation) associated with the monomial $z^{\alpha}$ (with coefficient -1 ), and

- in the row (or equation) associated with the monomial $z^{\alpha+A_{k}}$ (with coefficient +1 ).

(d) The right-hand side $r$ in the definition of $\Omega$ is integral. Therefore, as M is totally unimodular, whenever $\Omega \neq \emptyset$ each vertex of $\Omega$ is integral.

\subsection{Proof of Theorem 2.1}

(i) $\Rightarrow$ (ii) This follows directly from Proposition 2.2(b) and the fact that (2.1) holds if and only if $\Omega \neq \varnothing$.

(ii) $\Rightarrow$ (i) Suppose (2.1) holds for some polynomials $\left\{Q_{j}\right\} \subset \mathbb{R}\left[z_{1}, \ldots, z_{m}\right]$. Then $\Omega \neq \emptyset$ and so pick any vertex $\widehat{q}$ of $\Omega$. By Proposition 2.2(d), $\widehat{q} \in \Omega \cap \mathbb{Z}^{n s}$ and, by Proposition 2.2(a), $x:=\mathrm{E} \widehat{q} \in \mathrm{P} \cap \mathbb{Z}^{n}$, that is, $A x=b$ and $x \in \mathbb{N}^{n}$. 
From Proposition 2.2(b) and its proof, one sees that $q$ is a disaggregation of $x \in \mathbb{N}^{n}$. Indeed, if we write $q=\left(q_{1}, \ldots, q_{n}\right)$, then each $q_{j}$ has exactly $x_{j}$ nontrivial entries, all equal to 1 . So $q$ is not a lifting of $x$ as in the lift-and-project procedures described in [4]. In the latter, $x$ is part of the vector $q$ in the augmented space, and is obtained by projection of $q$.

\section{Main Result}

We first prove our results in the case $A \in \mathbb{N}^{m \times n}$ and then in Section 3.3, we show that the general case $A \in \mathbb{Z}^{m \times n}$ reduces to the former by adding one variable and one contraint to the original problem.

So let $A \in \mathbb{N}^{m \times n}, b \in \mathbb{N}^{m}$, and with no loss of generality we may and will assume that every column of $A$ has at least one nonzero entry, in which case $\mathrm{P}$ in (2.4) is a polytope.

Recall that with every solution $0 \leq q \in \mathbb{R}^{n s}$ of the linear system $\mathrm{M} q=r$ in (2.3) we may associate a set of polynomials $\left\{Q_{j}\right\} \subset \mathbb{R}\left[z_{1}, \ldots, z_{m}\right]$, with nonnegative coefficients, such that (2.1) is satisfied, and conversely to such a set of polynomials $\left\{Q_{j}\right\}$ with nonnegative coefficients, is associated a vector $0 \leq q \in \mathbb{R}^{n s}$ that satisfies (2.3). In fact, $q=\left\{q_{j \alpha}\right\}$ is the vector of coefficients of the polynomials $Q_{j}$ 's in the (usual) basis of monomials.

\subsection{An Equivalent Linear Program}

We now consider the integer program $\mathbb{P}$. For every $c \in \mathbb{R}^{n}$ let $\widehat{c} \in \mathbb{R}^{n s}$ be defined as

$$
\widehat{c}^{\prime}=\left({\widehat{c_{1}}}^{\prime}, \ldots,{\widehat{c_{n}}}^{\prime}\right) \quad \text { with } \quad \widehat{c}_{j}^{\prime}=c_{j}(1, \ldots, 1) \in \mathbb{R}^{s}, \quad \forall j=1, \ldots, n .
$$

Equivalently, $\widehat{c}^{\prime}=c^{\prime} \mathrm{E}$ with $\mathrm{E}$ as in (2.6). It also follows that $\widehat{c}^{\prime} q=c^{\prime} x$ whenever $x=\mathrm{E} q$. As a consequence of Theorem 2.1 we immediately obtain

Corollary 3.1. Let $A \in \mathbb{N}^{m \times n}, b \in \mathbb{N}^{m}, c \in \mathbb{R}^{n}$ be given. Let $\mathrm{M} \in \mathbb{Z}^{p \times n s}, r \in \mathbb{Z}^{p}$, and $\mathrm{E} \in \mathbb{N}^{n \times n s}$ be as in (2.3) and (2.6), respectively.

(a) The integer program

$$
\mathbb{P} \rightarrow \max _{x}\left\{c^{\prime} x \mid A x=b ; x \in \mathbb{N}^{n}\right\}
$$

has same optimal value as the linear program

$$
\mathbb{Q} \rightarrow \max _{q \in \mathbb{R}^{n s}}\left\{\widehat{c}^{\prime} q \mid \mathrm{M} q=r ; q \geq 0\right\}
$$

(including the case $-\infty$ ).

(b) In addition, let $q^{*} \in \mathbb{R}^{n s}$ be a vertex of $\Omega$ in (2.5), an optimal solution of the linear program $\mathbb{Q}$. Then $x^{*}:=\mathrm{E} q^{*} \in \mathbb{N}^{n}$ and $x^{*}$ is an optimal solution of the integer program $\mathbb{P}$. 
Proof. Let $\max \mathbb{P}$ and $\max \mathbb{Q}$ denote the respective optimal values of $\mathbb{P}$ and $\mathbb{Q}$. We first treat the case $-\infty \cdot \max \mathbb{P}=-\infty$ only if $\mathrm{P} \cap \mathbb{Z}^{n}=\emptyset$. However, then $\Omega=\emptyset$ as well, which in turn implies $\max \mathbb{Q}=-\infty$. Indeed, by Theorem 2.1, if $\mathrm{P} \cap \mathbb{Z}^{n}=\emptyset$, i.e., if $A x=b$ has no solution $x \in \mathbb{N}^{n}$, then one cannot find polynomials $\left\{\mathbb{Q}_{j}\right\} \subset \mathbb{R}\left[z_{1}, \ldots, z_{m}\right]$ with nonnegative coefficients that satisfy (2.1). Therefore, from the definition of $\Omega$, if $\Omega \neq \emptyset$ one would have a contradiction.

Conversely, if $\Omega=\emptyset$ (so that $\max \mathbb{Q}=-\infty$ ), then by definition of $\Omega$, one cannot find polynomials $\left\{\mathbb{Q}_{j}\right\} \subset \mathbb{R}\left[z_{1}, \ldots, z_{m}\right]$ with nonnegative coefficients that satisfy (2.1). Therefore, by Theorem 2.1, $A x=b$ has no solution $x \in \mathbb{N}^{n}$ which in turn implies $\max \mathbb{P}=-\infty$, i.e., $\mathrm{P} \cap \mathbb{Z}^{n}=\emptyset$.

In the case when $\max \mathbb{P} \neq-\infty$, we necessarily have $\max \mathbb{P}<\infty$ because the convex polyhedron $\mathrm{P}$ is compact. Next, consider a feasible solution $q \in \Omega$ of $\mathbb{Q}$. From Proposition 2.2(a) $x:=\mathrm{E} q \in \mathrm{P}$. Therefore, as $x$ is bounded then so is $\mathrm{E} q$, which, in view of definition (2.6) of E, also implies that $q$ is bounded. Hence $\Omega$ is compact which in turn implies that the optimal value of $\mathbb{Q}$ is finite and attained at some vertex $q^{*}$ of $\Omega$.

Now, let $x^{*} \in \mathbb{N}^{n}$ be an optimal solution of $\mathbb{P}$. By Proposition 2.2(b) there exists some $q \in \Omega$ with $\mathrm{E} q=x^{*}$. From definition (3.1) of the vector $\widehat{c}$ we have

$$
\widehat{c}^{\prime} q=c^{\prime} \mathrm{E} q=c^{\prime} x^{*}
$$

which implies $\max \mathbb{Q} \geq \max \mathbb{P}$.

On the other hand, let $q^{*} \in \Omega$ be a vertex of $\Omega$, an optimal solution of $\mathbb{Q}$. By Proposition 2.2(d), $q^{*} \in \Omega \cap \mathbb{Z}^{n s}$ and by Proposition 2.2(a), $x:=\mathrm{E} q^{*} \in \mathrm{P} \cap \mathbb{Z}^{n}$, that is, $x \in \mathbb{N}^{n}$ is a feasible solution of $\mathbb{P}$. Again, from definition (3.1) of the vector $\widehat{c}$ we have

$$
c^{\prime} x=c^{\prime} \mathrm{E} q^{*}=\widehat{c}^{\prime} q^{*},
$$

which, in view of $\max \mathbb{P} \leq \max \mathbb{Q}$, implies $\max \mathbb{P}=\max \mathbb{Q}$, and $x \in \mathbb{N}^{n}$ is an optimal solution of $\mathbb{P}$. This completes the proof of (a) and (b).

Remark 3.2. Let $b^{*}:=\sum_{j} b_{j}-\min _{k} \sum_{j=1}^{m} A_{j k}$ and $a:=\max _{k} \sum_{j=1}^{m} A_{j k}$. From the discussion immediately after Theorem $2.1, \mathrm{M} \in \mathbb{Z}^{p \times n s}$ where

$$
p=\left(\begin{array}{c}
m+b^{*}+a \\
b^{*}+a
\end{array}\right)=p_{1}(m) \quad \text { and } \quad s=\left(\begin{array}{c}
m+b^{*} \\
b^{*}
\end{array}\right)=p_{2}(m) .
$$

The polynomial $m \mapsto p_{1}(m)$ has degree $b^{*}+a$ whereas the polynomial $m \mapsto p_{2}(m)$ has degree $b^{*}$. Moreover, all the entries of $\mathrm{M}, r$ are $0, \pm 1$. Let $\mathcal{M}$ be the class of integer programs $\mathbb{P}$ with $A \in \mathbb{N}^{m \times n}, b \in \mathbb{N}^{m}$, and where, uniformly in $\mathbb{P} \in \mathcal{M}$,

- the column sums of $A$ are bounded (i.e., $\sup _{k} \sum_{j} A_{j k}$ is bounded), and

$-\sum_{j} b_{j}$ is bounded,

so that $a$ and $b^{*}$ above are bounded, uniformly in $\mathbb{P} \in \mathcal{M}$. Then one may solve the integer programs $\mathbb{P}$ of the class $\mathcal{M}$ in time polynomial in the problem size, because it suffices to solve the linear program $\mathbb{Q}$ which has $p_{1}(m)$ constraints and $n p_{2}(m)$ variables. One may consider this result as a dual counterpart of the known result which states that integer programs are solvable in time polynomial in the problem size when the dimension $n$ 
is fixed. (A dual counterpart would not be that integer programs are solvable in time polynomial in the problem size when the number of constraints $m$ is fixed. Just think of the knapsack problem where $m=1$.)

\subsection{The Integer Hull}

We are now interested in describing the integer hull $\mathrm{P}_{1}$ of $\mathrm{P}$, i.e., the convex hull of $\mathrm{P} \cap \mathbb{Z}^{n}$

Theorem 3.3. Let $A \in \mathbb{N}^{m \times n}, b \in \mathbb{N}^{m}$, and let $\mathrm{E} \in \mathbb{N}^{n \times n s}, \mathrm{M} \in \mathbb{Z}^{p \times n s}, r \in \mathbb{Z}^{p}$ be as in (2.6) and (2.3), respectively. Let $\left\{\left(u^{k}, v^{k}\right)\right\}_{k=1}^{t} \subset \mathbb{R}^{n \times p}$ be a (finite) set of generators of the convex cone $C \subset \mathbb{R}^{n \times p}$ defined by

$$
C:=\left\{(u, v) \in \mathbb{R}^{n \times p} \mid \mathrm{E}^{\prime} u+\mathrm{M}^{\prime} v \geq 0\right\} .
$$

(a) The integer hull $\mathrm{P}_{1}$ of $\mathrm{P}$ is the convex polyhedron defined by the linear constraints

$$
\left\langle u^{k}, x\right\rangle+\left\langle v^{k}, r\right\rangle \geq 0, \quad \forall k=1, \ldots, t,
$$

or, equivalently,

$$
\mathrm{P}_{1}:=\left\{x \in \mathbb{R}^{n} \mid U x \geq u\right\},
$$

where the matrix $U \in \mathbb{R}^{t \times n}$ has row vectors $\left\{u^{k}\right\}$, and the vector $u \in \mathbb{R}^{t}$ has coordinates $u_{k}=\left\langle-v^{k}, r\right\rangle, k=1, \ldots, t$.

(b) Equivalently $\mathrm{P}_{1}=\mathrm{E}(\Omega)$.

Proof. (a) Given $x \in \mathbb{R}^{n}$, consider the following linear system:

$$
\left\{\begin{array}{l}
\mathrm{E} q=x, \\
\mathrm{M} q=r \\
q \geq 0,
\end{array}\right.
$$

where M, E are defined in (2.3) and (2.6), respectively. Invoking the celebrated Farkas lemma (see, e.g., [7]), system (3.7) has a solution $q \in \mathbb{R}^{n s}$ if and only if (3.5) holds.

Therefore, let $x \in \mathbb{R}^{n}$ satisfy $U x \geq u$ with $U, u$ as in (3.6). By the Farkas lemma, system (3.7) has a solution $q \in \mathbb{R}^{n s}$, that is, $\mathrm{M} q=r, q \geq 0$, and $x=\mathrm{E} q$. As $q \in \Omega$ and $\Omega$ is compact, $q$ is a convex combination $\sum_{k} \gamma_{k} \widehat{q}^{k}$ of the vertices $\left\{\widehat{q}^{k}\right\}$ of $\Omega$. By Proposition 2.2(d), (a), for each vertex $\widehat{q}^{k}$ of $\Omega$ we have $\widehat{x}^{k}:=\mathrm{E} \widehat{q}^{k} \in \mathrm{P} \cap \mathbb{Z}^{n}$. Therefore,

$$
x=\mathrm{E} q=\sum_{k} \gamma_{k} \mathrm{E} \widehat{q}^{k}=\sum_{k} \gamma_{k} \widehat{x}^{k},
$$

that is, $x$ is a convex combination of points $\widehat{x}^{k} \in \mathrm{P} \cap \mathbb{Z}^{n}$, i.e., $x \in \mathrm{P}_{1}$; hence $\{x \in$ $\left.\mathbb{R}^{n} \mid U x \geq u\right\} \subseteq \mathrm{P}_{1}$.

Conversely, let $x \in \mathrm{P}_{1}$, i.e., $x \in \mathbb{R}^{n}$ is a convex combination $\sum_{k} \gamma_{k} \widehat{x}^{k}$ of points $\widehat{x}^{k} \in \mathrm{P} \cap \mathbb{Z}^{n}$. By Proposition 2.2(b), for each $k, \widehat{x}^{k}=\mathrm{E} q^{k}$ for some vector $q^{k} \in$ $\Omega \cap \mathbb{Z}^{n s}$. Therefore, as each $\left(\widehat{x}^{k}, q^{k}\right)$ satisfies (3.7), then so does their convex combination 
$(x, q):=\sum_{k} \gamma_{k}\left(\widehat{x}^{k}, q^{k}\right)$. By the Farkas lemma again, we must have $U x \geq u$, and so $\mathrm{P}_{1} \subseteq\left\{x \in \mathbb{R}^{n} \mid U x \geq u\right\}$, which completes the proof.

(b) This follows directly from (a) and

$$
\mathrm{E}(\Omega)=\left\{x \in \mathbb{R}^{n} \mid x=\mathrm{E} q ; \mathrm{M} q=r ; q \geq 0\right\} .
$$

Observe that the convex cone $C$ in (3.4) of Theorem 3.3 is defined explicitly in terms of the initial data $A$, and with no calculation. Indeed, the matrix M in (2.3) is easily obtained from $A$ and $\mathrm{E}$ is explictly given in (2.6). Thus, the interest of Theorem 3.3 is that we obtain an algebraic characterization (3.6) of $\mathrm{P}_{1}$ via generators of a cone $C$ simply related to $A$.

From the proof of Theorem 3.3, every element $(u, v)$ of the cone $C$ produces a valid inequality for $\mathrm{P}_{1}$, and, clearly, all strong valid inequalities can be obtained from generators of $C$.

Next suppose that for some $a \in \mathbb{R}^{n}, w \in \mathbb{R}$, we want to test whether $a^{\prime} x \geq w$ is a valid inequality. If there is some $v \in \mathbb{R}^{p}$ such that $\mathrm{M}^{\prime} v \geq-\mathrm{E}^{\prime} a$ and $-v^{\prime} r \geq w$, then, indeed, $a^{\prime} x \geq w$ is a valid inequality. In fact, $w$ can be improved to $\tilde{w}$ with

$$
\tilde{w}:=\max _{v}\left\{-v^{\prime} r \mid \mathrm{M}^{\prime} v \geq-\mathrm{E}^{\prime} a\right\} .
$$

\subsection{The General Case $A \in \mathbb{Z}^{m \times n}$}

In this section we consider the case where $A \in \mathbb{Z}^{m \times n}$, that is, $A$ may have negative entries. We assume that the convex polyhedron $\mathrm{P} \subset \mathbb{R}^{n}$, defined in (2.4), is compact.

Let $\alpha \in \mathbb{N}^{n}, \beta \in \mathbb{N}$ be such that for all $j=1, \ldots, m$,

$$
\widehat{b}_{j}:=b_{j}+\beta \geq 0, \quad \widehat{A}_{j k}:=A_{j k}+\alpha_{k} \geq 0, \quad k=1, \ldots, n .
$$

As $\mathrm{P}$ is compact we have

$$
\max _{x \in \mathbb{N}^{n}}\left\{\sum_{j=1}^{n} \alpha_{j} x_{j} \mid A x=b\right\} \leq \max _{x \in \mathbb{R}^{n} ; x \geq 0}\left\{\sum_{j=1}^{n} \alpha_{j} x_{j} \mid A x=b\right\}=: \rho^{*}(\alpha)<\infty .
$$

Given $\alpha \in \mathbb{N}^{n}$, the scalar $\rho^{*}(\alpha)$ is easily calculated by solving an LP problem. Note that we can choose $\beta \in \mathbb{N}$ as large as desired. Therefore, choose $\rho^{*}(\alpha) \leq \beta \in \mathbb{N}$. Let $\widehat{A} \in \mathbb{N}^{m \times n}, \widehat{b} \in \mathbb{N}^{m}$ be as in (3.9) with $\beta \geq \rho^{*}(\alpha)$.

The feasible solutions $x \in \mathbb{N}^{n}$ of $A x=b$, i.e., the points of $\mathrm{P} \cap \mathbb{Z}^{n}$, are in one-to-one correspondance with the solutions $(x, u) \in \widehat{\mathrm{P}} \cap \mathbb{Z}^{n+1}$ where $\widehat{\mathrm{P}} \subset \mathbb{R}^{n+1}$ is the convex polytope

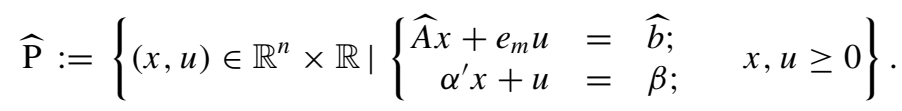

Indeed, if $x \in \mathrm{P} \cap \mathbb{Z}^{n}$, i.e., $A x=b$ with $x \in \mathbb{N}^{n}$, then

$$
A x+e_{m} \sum_{j=1}^{n} \alpha_{j} x_{j}-e_{m} \sum_{j=1}^{n} \alpha_{j} x_{j}=b+(\beta-\beta) e_{m},
$$


or, equivalently,

$$
\widehat{A} x+\left(\beta-\sum_{j=1}^{n} \alpha_{j} x_{j}\right) e_{m}=\widehat{b},
$$

and thus, as $\beta \geq \rho^{*}(\alpha) \geq \alpha^{\prime} x$, letting $u:=\beta-\alpha^{\prime} x \in \mathbb{N}$, yields $(x, u) \in \widehat{\mathrm{P}} \cap \mathbb{Z}^{n+1}$. Conversely, let $(x, u) \in \widehat{\mathrm{P}} \cap \mathbb{Z}^{n+1}$. Using the definitions of $\widehat{A}$ and $\widehat{b}$, it then follows immediately that

$$
A x+e_{m} \sum_{j=1}^{n} \alpha_{j} x_{j}+u e_{m}=b+\beta e_{m}, \quad \sum_{j=1}^{n} \alpha_{j} x_{j}+u=\beta,
$$

so that $A x=b$ with $x \in \mathbb{N}^{n}$, i.e., $x \in \mathrm{P} \cap \mathbb{Z}^{n}$. In other words,

$$
x \in \mathrm{P} \cap \mathbb{Z}^{n} \Leftrightarrow\left(x, \beta-\alpha^{\prime} x\right) \in \widehat{\mathrm{P}} \cap \mathbb{Z}^{n+1} .
$$

The convex polytope $\widehat{\mathrm{P}}$ can be written

$$
\widehat{\mathrm{P}}:=\left\{(x, u) \in \mathbb{R}^{n+1} \mid B\left[\begin{array}{l}
x \\
u
\end{array}\right]=(\widehat{b}, \beta) ; x, u \geq 0\right\},
$$

with

$$
B:=\left[\begin{array}{ccc}
\widehat{A} & \mid & e_{m} \\
- & - \\
\alpha^{\prime} & \mid & 1
\end{array}\right] .
$$

As $B \in \mathbb{N}^{(m+1) \times(n+1)}$, we are back to the case analyzed in Sections 3.1 and 3.2.

In particular, the integer program $\mathbb{P} \rightarrow \max \left\{c^{\prime} x \mid A x=b ; x \in \mathbb{N}^{n}\right\}$ is equivalent to the integer program

$$
\widehat{\mathbb{P}} \rightarrow \max \left\{c^{\prime} x \mid B\left[\begin{array}{l}
x \\
u
\end{array}\right]=\left[\begin{array}{l}
\widehat{b} \\
\beta
\end{array}\right] ;(x, u) \in \mathbb{N}^{n} \times \mathbb{N}\right\} .
$$

Hence, Theorem 2.1, Proposition 2.2, Corollary 3.1, and Theorem 3.3 are still valid with $B \in \mathbb{N}^{(m+1) \times(n+1)}$ in lieu of $A \in \mathbb{N}^{m \times n},(\widehat{b}, \beta) \in \mathbb{N}^{m} \times \mathbb{N}$ in lieu of $b \in \mathbb{N}^{m}$, and $\widehat{\mathrm{P}} \subset \mathbb{R}^{n+1}$ in lieu of $\mathrm{P} \subset \mathbb{R}^{n}$.

So again, as in previous sections, the polytope $\widehat{\Omega}$ associated with $\widehat{P}$ is explicitly defined from the initial data $A$, because $\widehat{A}$ is simply defined from $A$ and $\alpha$. In turn, as the convex cone $C$ in Theorem 3.3 is also defined explicitly from $A$ via $\mathrm{M}$, again one obtains a simple characterization of the integer hull $\widehat{\mathrm{P}}_{1}$ of $\widehat{\mathrm{P}}$ via the generators of $C$.

If we are now back to the initial data $A, b$, then $\mathrm{P}_{1}$ is easily obtained from $\widehat{\mathrm{P}}_{1}$. Indeed, by Theorem 3.3, let

$$
\widehat{\mathrm{P}}_{1}=\left\{(x, u) \in \mathbb{R}^{n+1} \mid\left\langle w^{k}, x\right\rangle+\delta^{k} u \geq \rho^{k} ; k=1, \ldots, t\right\},
$$

for some $\left\{\left(w^{k}, \delta^{k}\right) \in \mathbb{R}^{n} \times \mathbb{R}\right\}_{k=1}^{t}$, and some $t \in \mathbb{N}$. Then from (3.11) it immediately follows that

$$
\mathrm{P}_{1}=\left\{x \in \mathbb{R}^{n} \mid\left\langle w^{k}-\delta^{k} \alpha, x\right\rangle \geq \rho^{k}-\beta \delta^{k} ; k=1, \ldots, t\right\} .
$$




\section{4. $0-1$ Integer Programs}

The extension to $0-1$ integer programs

$$
\max _{x}\left\{c^{\prime} x \mid A x=b ; x \in\{0,1\}^{n}\right\}
$$

is straightforward by considering the equivalent integer program

$$
\max _{x, u}\left\{c^{\prime} x \mid A x=b ; x_{j}+u_{j}=1, \forall j=1, \ldots, n ; \quad(x, u) \in \mathbb{N}^{n} \times \mathbb{N}^{n}\right\},
$$

which is an integer program in the form (1.1). However, the resulting linear equivalent program $\mathbb{Q}$ of Corollary 3.1 is now more complicated. For instance, if $A \in \mathbb{N}^{m \times n}$, then $q \in \mathbb{R}^{2 n s}$ and $s$ is now the dimension of the vector space of polynomials in $n+m$ variables and of degree at most $n+\sum_{j} b_{j}$.

\section{Conclusion}

We have presented an explicit algebraic characterization of the integer hull $\mathrm{P}_{1}$ of a convex polytope $\mathrm{P} \subset \mathbb{R}^{n}$. Indeed, the defining hyperplanes of $\mathrm{P}_{1}$ are obtained from the generators of a convex cone whose description is obtained from the data $A, b$ with no calculation. Of course, and as expected, this convex cone is in a space of large dimension (exponential in the problem size). However, this structural result shows that all strong valid inequalities can be obtained in this manner. Therefore, we hope this result is helpful in deriving strong valid inequalities, or in validating some candidate inequalities, at least for some specific polytopes $P$.

\section{Acknowledgment}

The author is indebted to an anonymous referee for several helpful remarks improving the original version. In particular, he suggested the present proof of Theorem 2.1 which does not rely on the $\mathbb{Z}$-transform machinery used in [3].

\section{References}

1. G. Cornuejols and Y. Li, Elementary closures for integer programs, Oper. Res. Lett. 28 (2001), 1-8.

2. R. Jeroslow, An introduction to the theory of cutting-planes, Ann. Discrete Math. 5 (1979), 71-95.

3. J.B. Lasserre, A discrete Farkas lemma, Discrete Appl. Math., to appear.

4. M. Laurent, A comparison of the Sherali-Adams, Lovász-Schrijver and Lasserre relaxations for 0-1 programming, Math. Oper. Res. 28 (2003), 470-496.

5. E.W. Mayr and A.R. Meyer, The complexity of the word problems for commutative semigroups and polynomial ideals, Adv. in Math. 46 (1982), 305-329.

6. G.L. Nemhauser and L.A. Wolsey, Integer and Combinatorial Optimization, Wiley, Chichester, 1988.

7. A. Schrijver, Theory of Linear and Integer Programming, Wiley, Chichester, 1986.

8. L.A. Wolsey, Integer Programming, Wiley, New York, 1998.

Received February 12, 2003, and in revised form April 14, 2003, and April 25, 2003.

Online publication December 19, 2003. 